\title{
Polarity dependent properties of GaN layers grown by hydride vapor phase epitaxy on GaN bulk crystals
}

\author{
F. Tuomisto ${ }^{*}, 1,5$, T. Suski ${ }^{1}$, H. Teisseyre ${ }^{1}$, M. Krysko ${ }^{1}$, M. Leszczynski ${ }^{1}$, B. Lucznik ${ }^{1}$, \\ I. Grzegory ${ }^{1}$, S. Porowski ${ }^{1}$, D. Wasik ${ }^{2}$, A. Witowski ${ }^{2}$, W. Gebicki ${ }^{3}$, P. Hageman ${ }^{4}$, \\ and K. Saarinen ${ }^{5}$ \\ 1 High Pressure Research Center, Unipress, Polish Academy of Sciences, 01-142 Warsaw, Poland \\ 2 Institute of Experimental Physics, Warsaw University, Hoza 69, Warsaw, Poland \\ 3 Warsaw University of Technology, Koszykowa 75, 00-662 Warsaw, Poland \\ 4 NSRIM, University of Nijmegen, Toernooiveld 1, 6525 ED, Nijmegen, The Netherlands \\ 5 Laboratory of Physics, Helsinki University of Technology, P.O. Box 1100, 02015 HUT, Finland
}

Received 1 April 2003, accepted 24 June 2003

Published online 7 October 2003

PACS 71.55.Eq, 78.30.Fs, 78.55.Et, 78.70.Bj, 81.15.Kk

Hydride vapor phase epitaxy (HVPE) was used to grow nominally undoped 30-160 $\mu \mathrm{m}$ thick GaN layers on both the $\mathrm{Ga}$ and $\mathrm{N}$ polar sides of high pressure grown single crystal GaN substrates. Measurements of photoluminescence, infrared reflectivity, $\mu$-Raman scattering and positron annihilation were performed on the samples. The obtained characteristics are strongly dependent on the growth polarity. The material grown on the $\mathrm{N}$ polar side has identical properties with the substrate bulk GaN crystals, i.e. high free electron, impurity and point defect concentrations. In the layers grown on the Ga polar side, these concentrations are very low. The results show that the structural and optical properties of homoepitaxial GaN layers grown by HVPE are homogeneous across the layer thickness, which is in contrast to the heteroepitaxial HVPE GaN layers.

A significant influence of the gallium and nitrogen polar directions $(\langle 00.1\rangle$ and $\langle 00 . \overline{1}\rangle)$ on the oxygen incorporation in wurtzite gallium nitride $(\mathrm{GaN})$ has been theoretically predicted by Zywietz et al. [1]. A higher density of donors has been observed experimentally on the $\mathrm{N}$ polar surface by secondary ion mass spectroscopy (SIMS). In addition, a gradient of the free carrier concentration along the $c$-axis has been found $[2,3]$. Thin heteroepitaxial $\mathrm{GaN}$ overlayers grown on sapphire $\left(\mathrm{Al}_{2} \mathrm{O}_{3}\right)$ by molecular beam epitaxy (MBE) [4] and metal-organic vapor phase epitaxy (MOVPE) [5] have also shown that the layer polarity has a significant impact on the oxygen concentration in the layer. However, the heteroepitaxial layers have a high dislocation density due to the lattice mismatch between the layer and the substrate. The high dislocation density can affect the impurity incorporation, diffusion, and point defect formation in the material. An ideal system for investigating the polarity dependent properties of the GaN overlayers, independent of the extended defect concentration, is thus the homoepitaxial layer grown on the dislocation-free high pressure grown GaN single crystal [6-8]. Moreover, with hydride vapor phase epitaxy (HVPE), thick layers can be grown within reasonably short times, and the impact of the layer thickness on the properties can be studied as in the case of heteroepitaxial HVPE $\mathrm{GaN}[9,10]$. The quality of the heteroepitaxial layers improves with layer thickness, implying that the physical properties of the layers vary along the $c$-axis.

\footnotetext{
${ }^{*}$ Corresponding author: current address: Laboratory of Physics, Helsinki University of Technology, P.O. Box 1100, 02015 HUT, Finland, e-mail: filip.tuomisto@hut.fi, Phone: +35894513144, Fax: +35894513116
} 
In this work, we investigate the optical and structural characteristics of thick HVPE GaN epilayers grown on facets of both $\mathrm{N}$ and Ga polarities of bulk GaN substrates. Our results show that the $\mathrm{N}$ polar layer has a high concentration of free electrons and $\mathrm{Ga}$ vacancies, indistinguishable from the substrate bulk GaN crystal. On the other hand, the Ga polar layer has excellent optical and structural properties together with free electron and Ga vacancy concentrations below the sensitivity limits of the experimental techniques used. The results support the theoretical prediction [1] on the influence of the growth polar direction on the oxygen incorporation. Moreover, the optical and structural properties of the homoepitaxial HVPE GaN layers do not depend on the layer thickness and are homogeneous along the $c$-axis.

The layers studied were grown on specially prepared $\mathrm{N}$ and Ga polarity surfaces of bulk GaN substrates. The achieved growth rates were $15-80 \mu \mathrm{m} / \mathrm{h}$. Details of the growth procedure can be found in Ref. [11]. The high crystallographic quality of the substrates, i.e. a density of dislocations as low as $10^{2} \mathrm{~cm}^{-2}$, is reproduced in the layers of both $\mathrm{Ga}$ and $\mathrm{N}$ polarities [12]. The optical properties of the layers were measured with infrared reflectivity (IR), $\mu$-Raman scattering and photoluminescence (PL) spectroscopies. We used positron annihilation measurements to probe the vacancy-type defects.

The free electron concentration was measured with IR experiments at room temperature with a Bruker IFS 113v FT-IR spectrometer at near-normal incidence. The reflectivity data was analyzed by fitting the theoretical reflectivity spectrum by the means presented in previous works on high pressure grown GaN single crystals [3, 13]. The IR spectra measured from the HVPE layer grown on the $\mathrm{N}$ and $\mathrm{Ga}$ polar sides are presented in Fig. 1a. The spectrum of the $\mathrm{N}$ polar layer is clearly dominated by the reflection from the free electron plasma. The IR spectrum of the Ga polar layer on the other hand is dominated by the absorption peak of the LO phonon.

We measured the homogeneity of the layers with $\mu$-Raman scattering experiments with a Dilor XY $800 \mu$-Raman spectrometer connected to a CCD-camera. The $514.5 \mathrm{~nm}$ line of a $400 \mathrm{~mW} \mathrm{Ar}^{+}$laser was used for excitation. The measurements were performed at room temperature and the measurement configuration was $x(z,-) \bar{x}$. Typical Raman spectra recorded from the HVPE layers are presented in Fig. 1b. The spectra were identical along the $c$-axes of the layers, thus showing their homogeneity. The $A_{1}(\mathrm{TO})$ and $E_{1}(\mathrm{TO})$ as well as the $E_{2}$ phonon modes are clearly visible in both spectra in Fig. $1 \mathrm{~b}$. In the Raman spectrum of the HVPE layer grown on the Ga side the $\mathrm{LPP}^{+}$mode frequency coincides with the $A_{1}(\mathrm{LO})$ mode frequency. In the $\mathrm{N}$ polar HVPE layer, where the electron plasma frequency is high, the $\mathrm{LPP}^{+}$mode frequency coincides with the plasma frequency [13] and cannot be seen in Fig. 1b, but it should appear at around $2300 \mathrm{~cm}^{-1}$.

The photoluminescence of the samples was measured at $10 \mathrm{~K}$ in the backscattering setup. The excitation was produced by the $325 \mathrm{~nm}$ line of a $2.5 \mathrm{~mW}$ He-Cd laser. Figure 2 shows the PL spectra
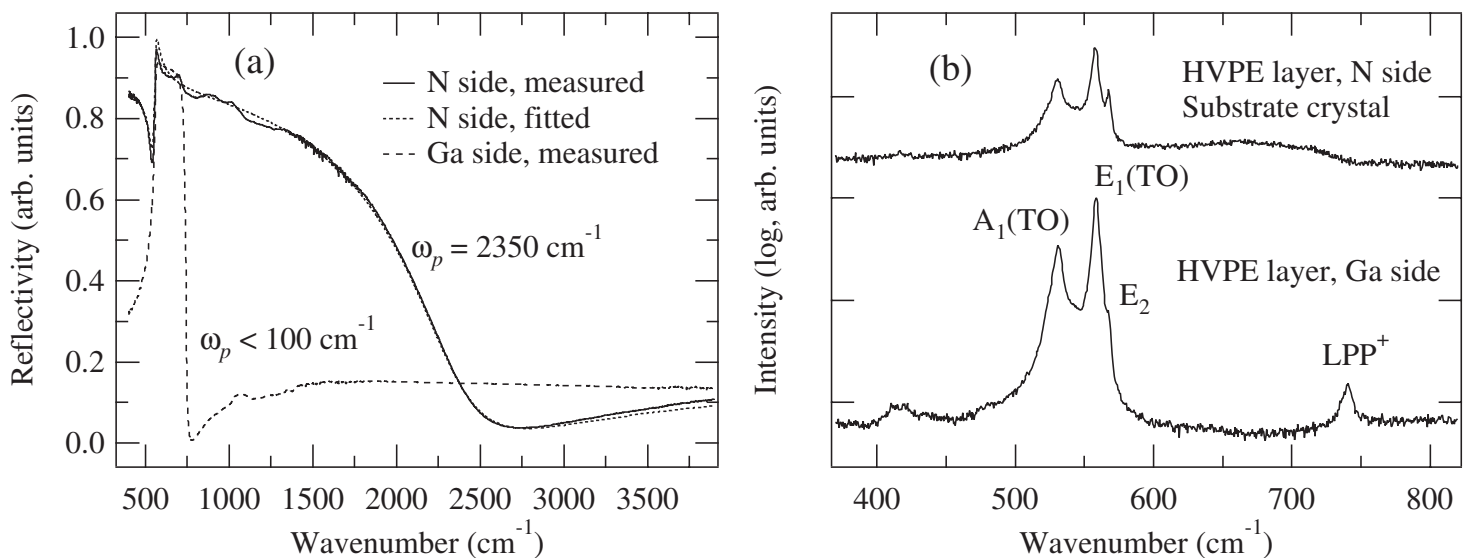

Fig. 1 a) Infrared reflectivity spectra measured from the $\mathrm{N}$ and Ga polar HVPE layers. The fitted theoretical spectrum is presented for the $\mathrm{N}$ polar layer. b) Raman spectra of the N and Ga polar HVPE layers. 


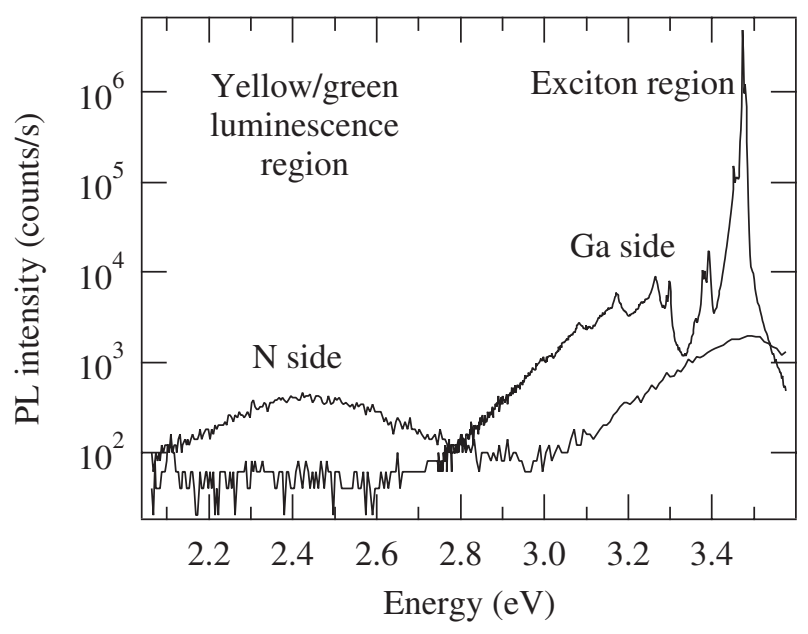

Fig. 2 Photoluminescence spectra of the Ga and $\mathrm{N}$ polar HVPE layers.

measured from the HVPE layers grown on the $\mathrm{N}$ and $\mathrm{Ga}$ polar sides of the single crystal substrate. The broad yellow/green PL peak is clearly visible in the spectrum of the N polar layer. On the other hand, the spectrum measured from the Ga polar layer is dominated by exciton and donor-acceptor pair (DAP) transition PL peaks and their satellites.

The vacancy concentrations in the layers were measured with positron lifetime experiments using a conventional fast-fast spectrometer in collinear geometry [14] with a time resolution of 250 ps. In this scheme the positron source $\left({ }^{22} \mathrm{Na}\right.$ deposited on $1.5 \mu \mathrm{m} \mathrm{Al}$ foil) is sandwiched between two identical sample pieces. The lifetime spectrum was analyzed as the sum of exponential decay components convoluted with the Gaussian resolution function of the spectrometer, after subtracting the constant background and annihilations in the source material. Figure 3 shows the positron lifetime measured from the HVPE layers grown on both sides of the single crystal. It is clearly seen that the average positron lifetime in the $\mathrm{N}$ polar layer is longer than that in the Ga polar layer.

Only one exponential lifetime component of $\tau_{\text {ave }}=161 \mathrm{ps}$ could be fitted to the lifetime spectrum of the Ga polar layer. Its value corresponds to the annihilation from the free state in the lattice [15]. Thus the HVPE layers grown on the Ga side can be considered to have a vacancy concentration

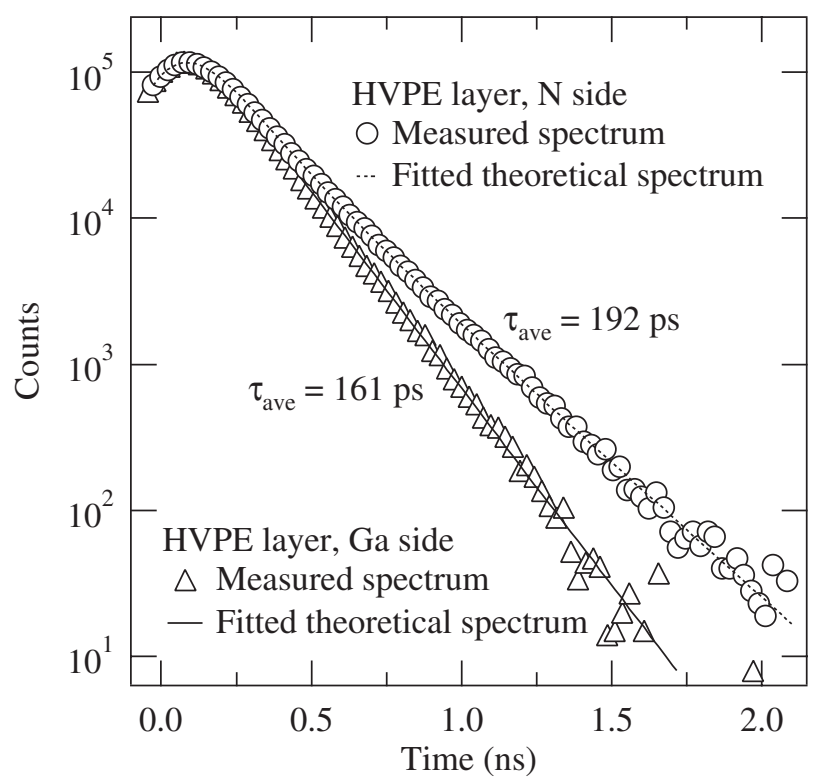

Fig. 3 Measured and fitted positron lifetime spectra in the HVPE GaN layers grown on the $\mathrm{N}$ and $\mathrm{Ga}$ polar sides of the single crystal $\mathrm{GaN}$ substrate. 
$\left[V_{\mathrm{Ga}}\right] \leq 10^{16} \mathrm{~cm}^{-3}$, i.e. below the sensitivity range. The longer average positron lifetime in the $\mathrm{N}$ polar layer indicates the presence of open-volume defects. Two lifetime components could be separated from the experimental positron lifetime spectrum, the longer lifetime component being $\tau_{2}=237 \pm 3$ ps. This lifetime can be attributed to the Ga vacancy [15]. From these results, the Ga vacancy concentration in the $\mathrm{N}$ polar HVPE layer can be estimated to be $\left[V_{\mathrm{Ga}}\right] \simeq 4 \times 10^{17} \mathrm{~cm}^{-3}$.

The free electron concentration in the HVPE layers of different polarity was calculated from the IR results, giving $N_{e} \simeq 7 \times 10^{19} \mathrm{~cm}^{-3}$ for the $\mathrm{N}$ polar layer and $N_{e} \leq 10^{17} \mathrm{~cm}^{-3}$ for the Ga polar layer. The $\mu$-Raman spectra measured at different depths support this result, and show that the free electron concentrations are homogeneous across the thickness of the layer. As in earlier studies [15], the yellow luminescence can be attributed to the deep acceptor levels formed by the Ga vacancies. The Ga vacancies have been shown to be stabilized by the residual oxygen impurities, which are present in the nominally undoped bulk GaN crystals [16].

In conclusion, all results suggest that a high $\left(\sim 10^{19} \mathrm{~cm}^{-3}\right)$ concentration of donors (likely oxygen) is present in the $\mathrm{N}$ polar layers and is responsible for the high free electron concentration. The observation of the DAP and exciton peaks in the photoluminescence spectrum of the Ga polar layer is a clear indication of a low free electron concentration and supports the IR and $\mu$-Raman results. These observations are in perfect agreement with the theoretical predictions [1] on the adsorption of oxygen and other impurities on the polar $\mathrm{Ga}$ and $\mathrm{N}$ faces of the wurtzite $\mathrm{GaN}$ crystal. In contrast to the heteroepitaxial HVPE GaN layers [9], the homoepitaxial HVPE layers are homogeneous and their characteristics do not depend on the layer thickness. Thus, since they exhibit extremely low dislocation density, it is suggestive to assume a contribution of the extended defects, mainly dislocations, to the point defect and donor concentration distribution in the heteroepitaxial HVPE GaN layers. The material grown on the $\mathrm{N}$ polar side of $\mathrm{GaN}$ single crystals has a high crystallographic quality but reproduces also the impurity, optical and point defect properties of the bulk GaN substrate. On the other hand, the layers grown on the Ga polar side combine the structural quality of the bulk crystal with very low impurity and point defect concentrations as well as excellent optical properties.

Acknowledgement The work in Warsaw was supported by the Project of European Commission DENIS, G5RD-CT-2001-00566.

\section{References}

[1] T. Zywietz, J. Neugebauer, and M. Scheffler, Appl. Phys. Lett. 54, 1695 (1999).

[2] P. Prystawko et al., phys. stat. sol. (b) 210, 437 (1998).

[3] E. Frayssinet et al., J. Cryst. Growth 230, 442 (2001).

[4] A. Ptak et al., Appl. Phys. Lett. 79, 2740 (2001).

[5] S. Chichibu et al., Appl. Phys. Lett. 78, 28 (2001).

[6] J. Karpinski, J. Jun, and S. Porowski, J. Cryst. Growth 66, 1 (1984).

[7] I. Grzegory et al., Acta Phys. Pol. A 100, Suppl. 57 (2001).

[8] M. Leszczynski et al., J. Cryst. Growth 169, 235 (1996).

[9] J. Oila et al., Appl. Phys. Lett. (in press).

[10] H. Morkoç, Mater. Sci. Eng. R 33, 135 (2001).

[11] M. Bockowski et al., J. Cryst. Growth 246, 194 (2002).

[12] Z. Liliental-Weber, J. Jasinski, and J. Washburn, J. Cryst. Growth 246, 259 (2002).

[13] P. Perlin et al., Appl. Phys. Lett. 67, 2524 (1995).

[14] K. Saarinen, P. Hautojärvi, and C. Corbel, in: Identification of Defects in Semiconductors, edited by M. Stavola (Academic Press, New York, 1998), p. 209.

[15] K. Saarinen et al., Phys. Rev. Lett. 79, 3030 (1997).

[16] J. Oila et al., Phys. Rev. B 63, 045205 (2001). 